\title{
The Virtual Team Member: Remote Engagement of Medical Students in COVID-19 Care
}

\author{
Cecilia Yoon ${ }^{1}$ (I) $\cdot$ Jihui Lee $^{1} \cdot$ Ericka Fong $^{1} \cdot$ Jennifer I. Lee ${ }^{1}$
}

Accepted: 27 September 2021 / Published online: 19 October 2021

(c) International Association of Medical Science Educators 2021

\begin{abstract}
Background Medical education abruptly changed in the setting of the COVID-19 pandemic, impacting experiential learning in clinical clerkship as medical students were removed from direct patient interactions and care team participation. Reconfiguring a hospital clinical rotation using virtual care platforms allowed students to re-engage in the clinical environment and actively participate in patient care.

Methods During the height of the pandemic, we implemented a 4-week "virtual team member" (VTM) inpatient medicine elective for medical students in their second year and above to participate in acute patient care during the height of the COVID-19 pandemic. Tasks included providing daily updates to patients and family members along and care coordination. Faculty experts in infectious disease, mental health, ethics, and patient safety incorporated weekly didactic video talks throughout the elective. Student feedback was obtained anonymously through pre-, mid-, and post-elective questionnaires. Results A total of 26 students enrolled in the two 4-week blocks, with $85 \%$ in the 2 nd year. Survey response rates for the pre, mid, and post-rotation questionnaires were $96 \%, 77 \%, 58 \%$ respectively. Of the 15 students who completed the post-survey, the majority strongly and somewhat agreed that the elective met expectations $(12 / 15,80 \%)$, was worthwhile $(14 / 15,93 \%)$ and met goals $(12 / 15,80 \%)$. Best parts of the elective most frequently cited by students were patient care and teamwork. Working remotely was the greatest challenge.

Conclusions Designing a virtual role for students successfully allowed students to re-engage in the acute care setting and connect with patients and participate in COVID-19 care.
\end{abstract}

Keywords Medical education $\cdot$ Covid pandemic $\cdot$ Virtual elective

\section{Introduction}

On March 17, 2020, the Association of American Medical Colleges (AAMC) strongly suggested a pause in clinical rotations for medical students during the COVID-19 pandemic [1]. For students, this resulted in loss of experiential learning and the opportunity to be part of a care team. It removed the next generation of physicians from active engagement in the largest global public health crisis of the century.

The cancellation of clinical rotations required educators to reassess the traditional learning paradigm. New York City, where our institution is located, emerged as an early epicenter of the COVID-19 pandemic with an executive order

Cecilia Yoon

cjy2001@med.cornell.edu

1 Department of Medicine, Weill Cornell Medicine/New York Presbyterian Hospital, 53 West 23rd street, 6th floor, New York, NY, USA in March 2020, closing non-essential business and banning non-essential gatherings of individuals. Alongside this, hospitals began postponing elective procedures and converting non-urgent ambulatory clinic visits to telehealth care, redeploying staff and resources in anticipation for surge of inpatients including need for ICU care and formation of COVID-19 unit. At Weill Cornell Medical College, clinical clerkships underway at the time of the pause transitioned to a remote learning format using recorded lectures and interactive case discussions to allow students to complete these courses. A COVID-19 response steering committee (CRSC) was convened to develop a curriculum to ensure continuing academic progress including requests for proposal to clinical department education leaders for real-time interactive telehealth experiences (RITEs) that followed institutional standard elective format alongside a required debrief and reflection sessions with a goal of designing credit-bearing remote activities during the projected 8-week pause of onsite clinical care [2]. 
Optimizing experiential learning without patient contact posed practical and logistical challenges but also an opportunity for innovation. With advancements in technology and electronic medical records (EMR) systems, telemedicine has been shown to be an effective medium to provide directed care with improved outcomes and satisfaction in the urgent care setting and the management of chronic conditions as highlighted by the rapid implementation during the COVID19 pandemic with delivery of specialty and subspecialty care including mental health [3-10].

There are many challenges to overcome when applying virtual learning platforms to the inpatient setting. Unlike pre-clerkship courses where transition to online learning formats has been less disruptive while meeting educational outcomes, preserving the value of clinical experiences would be difficult while physically distanced from patients and the care team. Throughout the pandemic, educators have collaborated with clinical faculty to create virtual educational activities in lieu of paused clinical clerkships such as medicine, surgery, and ophthalmology using combinations from self-study material to online didactic/case discussions and telehealth experiences with variable effect since the objective measures ranged from student specialty interest to knowledge [11-16]. Unpredictable changes in onsite team activities due to the uncertainties of COVID-19 care could lead to inconsistent student experiences in team engagement, supervision, and inclusion in the clinical decision-making process. Optimizing student engagement and experiential learning would require clearly defined and feasible role and responsibilities, focusing course objectives to meet student expectations, and ongoing feedback for continuous quality improvement.

The Front-line Care; Virtual Team Member elective was developed as a 4-week elective on the general medicine service and designed to fill a unique and crucial gap in the care of COVID-19 patients-providing longitudinal social support and bridging patient-team communications for persons under investigation (PUI) and COVID-19 positive patients hospitalized in strict isolation. Over 6 weeks, our institution's inpatient COVID-19 census peaked to nearly 500 patients, leaving medical teams contending to provide rapidly evolving clinical care to patients often at the expense of delivering timely updates and support to patients and their distanced families. There was a need to provide emotional support to COVID-19 patients and their families facing profound uncertainties and fear and keeping them informed of their medical plan. VTMs would fill that gap while being directly involved in the clinical care plans of this unique cohort. This report demonstrates the rapid implementation and feasibility of an inpatient virtual elective for medical students caring for SARS-CoV2-infected persons. This article details the elective's format and qualitative feedback from the medical students on the strengths and weaknesses of their experience. According to institutional review board (IRB) policies at our institution, this work met criteria for quality improvement activities to evaluate and improve a specific practice or program and was exempt from IRB review.

\section{Methods}

Our institution is composed of an 862-bed quaternary referral center and an affiliated 180-bed non-teaching community hospital in Manhattan. During late March 2020, ORs and PACUs were converted to ICUs wherever feasible. Nonmedical units including psychiatric wards were converted to medical units requiring expansion of the number of inpatient hospital medical teams. Teaching teams were composed of an attending, 1 resident, and 1 intern. Up to two physician assistants were paired with the attending on the non-teaching service. In addition, due to emerging observation of the post-COVID syndrome and need for further comprehensive chronic care, a 30-bed COVID-19 Recovery Unit managed by non-teaching teams was established.

In April 2020, clinician educators designed a 4-week virtual inpatient elective for medical students second year (MS2) and above to work as a VTM on a teaching or nonteaching hospitalist service taking care of admitted PUI and COVID-19-positive patients. Our elective was 1 of 4 RITE developed with a total capacity 38 students per 4-week block. The elective was offered from April 6 to May 1, 2020 (Block 1) and from May 4 to May 31, 2020 (Block 2). The learning objectives included (1) active engagement in frontline care of COVID-19-positive and PUI patients as a core member of the Hospital Medicine team; (2) expediting care through obtaining and relaying patient-related information to the team such as past medical and family histories, home medications; (3) participation in goals of care discussions led by the attending hospitalist with patients and their families. This course was graded pass/fail, in alignment with all elective offerings at our institution. Students registered for the VTM elective through their online curriculum portal. All students were given access to the inpatient EMR and the hospital HIPAA-compliant messaging platform to securely contact team members.

Prior to the start of each block, VTMs and their assigned attendings received orientation emails outlining their respective roles and expectations. They exchanged cell phone contact information and arranged a time for VTMs to call into morning rounds. We anticipated fluctuation in the daily schedule but outlined general expectations for the students to complete while on service (Fig. 1). Activities were added and adjusted based on patient and service needs.

The VTM would be available Monday through Friday and assigned up to five patients working with residents and 


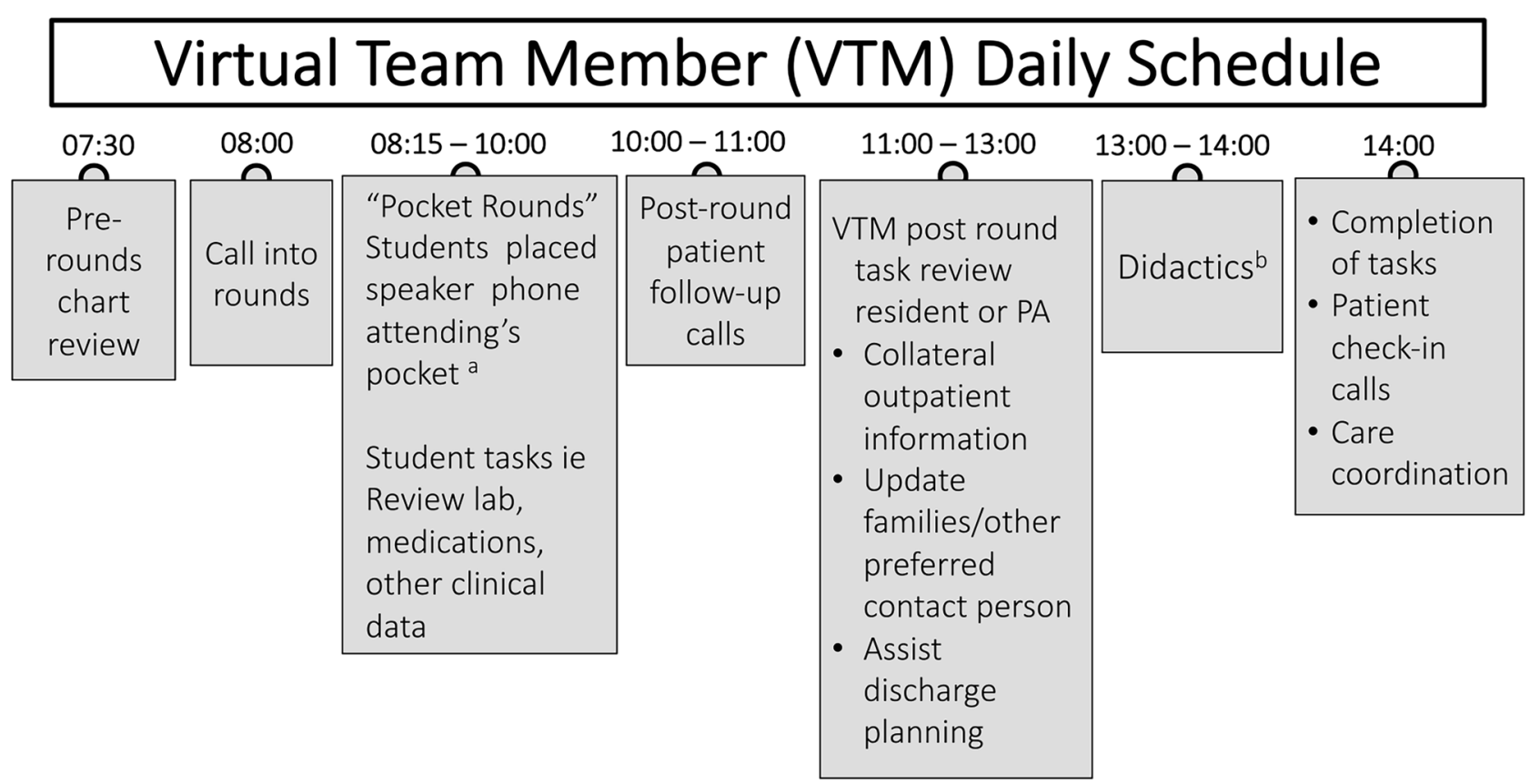

Fig. 1 Virtual COVID team member elective schedule (April-May 2020). attendings introduce the student to the patient if assent obtained to communicate with them and/or family member. Patients unable to speak or be heard legibly, clinically unstable, or high oxygen demand were excluded. ${ }^{\mathbf{b}}$ Video conferences with faculty experts took place once a week on the following topics: (1) SARS-CoV-2 and COVID-19: pathophysiology, epidemiology, clinical manage-

physician assistants under the hospitalist's supervision. The hospitalists assigned admitted patients to the VTM if the patients agreed to follow-up calls and were able to engage in conversation. Patients were excluded if they declined to participate and were determined by the attending to have unstable respiratory status or admitted to the intensive care unit. Students used hospital-approved phone interpreters to engage limited English proficiency patients.

VTMs began their day with pre-round electronic chart reviews on their assigned patients before calling into morning rounds. Patients received calls on their room landline or cell phone including video calls when available with a goal of twice daily check-ins arranged at the patient's convenience as an "appointment" to help orient them to time. Students were reminded to escalate to the primary team any concerns voiced by the patients or recognized by the students during the daily calls. Urgent issues were immediately communicated to the attending hospitalist and virtual psychiatrists for ongoing assessment. All tasks completed were documented in the EMR.

Video conferences with faculty experts took place once a week on the following topics: (1) SARS-CoV-2 and COVID19: pathophysiology, epidemiology, clinical management, experimental trials; (2) communication skills; (3) patient safety; (4) mental health primer; (5) medical ethics and ment, experimental trials; (2) communication skills; (3) patient safety; (4) mental health; (5) medical ethics. Other days, didactics were scheduled ad hoc based on the hospitalist or resident's availability. Attendings typically by themselves go into rooms of patients with COVID-19 and with their permission have students on speaker "Pocket-Rounds" where they can listen to the discussion and treatment plan in real time including goals of care

COVID-19. Students selected one QPS project to complete during their 4-week block: a mini-root cause analysis (RCA) of a medical error they identified during the course or standardizing COVID-19 patient-centered handoffs to improve rapid care transfers across settings (see Appendix 1). In addition, we held once weekly debrief sessions as requested by the CRSC to review students' experiences, reflections, and concerns. Feedback was used to rapidly adjust the course content and structure as needed.

\section{Survey}

With the incoming Class of 2018, our institution launched a new three-phased curriculum: condensed pre-clinical foundations coursework (Phase 1), core clinical clerkship starting in the middle of 2nd year (Phase 2), scholarship and advanced clinical skills with a focus on Area of Concentration project (Phase 3 ). Given that the elective was designed rapidly in 3 days to meet an emergent patient care need, we recognized that continuous quality improvement would be needed to optimize the elective experience to meet the educational needs of the students as well as remain within the level of clinical demand the students were able to meet. The surveys were a composite designed by elective leadership from several prior QI project assessment tools (see 
Appendix 2, full survey questions). The data informed rapid cycle testing through Plan-Do-Study-Act iterations to abandon, adapt, or adopt changes targeting areas of concern. We assessed the outcomes for the improvements and their overall elective experience by repeating the survey at the end of each elective block. The surveys were not intended to be a formal assessment of the course nor of the students' performance.

Students completed a voluntary anonymous survey at the beginning, at 2 weeks and at the end of each 4-week block. Since the elective was open to MS2 and above, we anticipated varying degrees of clinical experience and patient care exposure. The pre-elective survey was mainly qualitative and designed to gauge the students' baseline clinical background, completed clerkships, and expectations for enrolling in the elective. This information was used to tailor the collective responsibilities and expectations of the VTMs and their assigned attendings.

The post-elective survey assessed students' satisfaction using both qualitative (open-ended) and quantitative (5-point Likert scale) questions. Likert scale questions addressed three categories: experience, supervision, and goals. Each category consisted of 3 or 4 questions, and the 5-point Likert scale for those questions was "strongly disagree," "somewhat disagree," "neutral," "somewhat agree," and "strongly agree" (Table 1).

While the wording of the questions was different across the two questionnaires, the sentiment and context we intended to obtain from the open-ended free text qualitative questions were similar and could be used in direct comparison. In the pre-elective survey, students were asked about their goals (positive; "What are your goals for the VTM elective rotation?") and anticipated challenges (negative; "What do you anticipate to be the biggest challenges you will face on the VTM elective rotation?") about the VTM elective. Similarly, the post-elective survey asked about the best (positive; "What was the best part of the elective?") and the worst (negative; "What was the worst part of the elective?") part of the VTM elective. We anticipated that being the only team member to be offsite and working remotely would pose challenges of feelings of disengagement from the team and frustration with successfully contacting patients and hearing them clearly through obstacles (oxygen masks with portable HEPA filters whirling in the background). The 2-week mid-elective survey was used for internal quality control purposes only to optimize the students' time spent on various activities during their day. We used this information to provide tips on workflow such as the best times to call patients, use of phone translators, timing, and topics of video conferences.

\section{Data Analysis}

The authors (CJY, JIL, JL, EF) independently reviewed and manually extracted keywords from the responses to the qualitative questions from the pre-elective and post-elective surveys. Keywords identified by more than 2 authors were entered into an Excel spreadsheet. Sentiment and context of each keyword was taken into consideration in this process. Some keywords were grouped to construct a unique theme; for example, key words including "COVID care," "ICU care," and "Crisis" represented the theme "Taking care of patients." The theme "Working as a team" contained keywords such as "Contribute," "Responsibility," and "Communication." Distribution of the five identified themes for pre-elective expectations and post-elective feedback was compared using Fisher's exact test in order to form a qualitative assessment based on students' sentiments and

Table 1 Post-elective survey responses about students' overall experiences

\begin{tabular}{|c|c|c|c|c|c|c|}
\hline \multirow[t]{2}{*}{ Category } & \multirow[t]{2}{*}{ Survey question } & \multicolumn{5}{|c|}{$\begin{array}{l}\text { Responses } \\
N=15^{*}(\%)\end{array}$} \\
\hline & & $\begin{array}{l}\text { Strongly } \\
\text { disagree }\end{array}$ & $\begin{array}{l}\text { Somewhat } \\
\text { disagree }\end{array}$ & Neutral & Somewhat agree & Strongly agree \\
\hline \multirow[t]{4}{*}{ Experience } & The elective has met my expectations & $0(0)$ & $3(20)$ & $0(0)$ & $10(67)$ & $2(13)$ \\
\hline & The elective has been professionally worthwhile & $0(0)$ & $0(0)$ & $1(7)$ & $8(53)$ & $6(40)$ \\
\hline & The elective gave me sufficient practice and feedback & $0(0)$ & $1(7)$ & $3(20)$ & $8(53)$ & $3(20)$ \\
\hline & I felt included by the medical team & $0(0)$ & $0(0)$ & $2(13)$ & $8(53)$ & $5(33)$ \\
\hline \multirow[t]{3}{*}{ Supervision } & Leadership clearly explained my role and what was expected of me & $0(0)$ & $1(7)$ & $2(13)$ & $7(47)$ & $5(33)$ \\
\hline & Leadership encouraged me to share my own ideas and ask questions & $0(0)$ & $2(13)$ & $0(0)$ & $3(20)$ & $10(67)$ \\
\hline & Leadership was adequately accessible for my questions and/or concerns & $0(0)$ & $1(7)$ & $0(0)$ & $3(20)$ & $11(73)$ \\
\hline \multirow[t]{3}{*}{ Goals } & $\begin{array}{l}\text { This elective allowed me to participate in frontline clinical care as a } \\
\text { "virtual" and essential member of the care team }\end{array}$ & $0(0)$ & $0(0)$ & $3(20)$ & $8(53)$ & $4(27)$ \\
\hline & $\begin{array}{l}\text { This elective helped me master communication skills essential for } \\
\text { frontline care }\end{array}$ & $0(0)$ & $0(0)$ & $1(7)$ & $11(73)$ & $3(20)$ \\
\hline & $\begin{array}{l}\text { This elective allowed me to become a "virtual" companion and social } \\
\text { support for patients }\end{array}$ & $0(0)$ & $1(7)$ & $0(0)$ & $7(47)$ & $7(47)$ \\
\hline
\end{tabular}


impressions of their experience. The 5-point Likert scale responses to quantitative questions from post-elective survey were considered as categorical variables and summarized using frequencies and percentages. All analyses were done in R 3.6.1 (R Core Team, Vienna, Austria).

\section{Results}

A total of 26 students participated in the elective across both blocks. Eighty-five percent (22/26) were MS2. Forty percent (10/26) completed a Pediatrics or Internal Medicine clerkship prior to the elective. Survey response rates were $96 \%$ (25/26) for the pre-elective survey, $77 \%$ (20/26) for the mid-elective survey, and 58\% (15/26) for the postelective survey. The mid-elective survey results were used for internal quality improvement purposes only to help the elective leadership team to target and address issues during the elective.

We compared student responses to the pre-elective expectations and post-elective feedback (Fig. 2). The five overarching themes were listed in order of highest frequency: taking care of patients, working as a team, improving medical knowledge, supporting patients emotionally, and experiencing telemedicine. Among survey responders, "taking care of patients" and "working as a team" were both the highest reported expectation and "best part" of the elective, indicating that their pre-elective expectations were met by the course. Highlights of post-rotation students' comments include,

Feeling like I was able to make contributions during this crisis, learning medicine, and being connected with clinical role models
Being able to build a rapport and relationship with the family, and walk with them thru it ....I never got to work so closely with attendings during clerkships...

Similarly, students were asked to list concerns of starting a virtual inpatient rotation. Working remotely was the most frequently cited theme and echoed again in responses to "What was the worst part of the elective" on the postelective survey. Issues included inability to reach patients by phone, feeling disconnected from the onsite teams, and difficulty hearing conversations. Frequent team turnover and inconsistent expectations of the VTM were also listed as consequences of the rapidly evolving patient care demands of the inpatient teams during the COVID-19 crisis. Student comments included:

Not be able to physically interact with patients and perform physical exams.

Experience varied significantly by teams and attendings, and there were times where I did not feel like I was part of the team (listened into rounds but had no responsibilities).

Weekly didactic topics focused on relevant issues encountered during the care of COVID-19-infected patients. Attendance was mandatory in all the didactic and debrief sessions, and QPS projects were completed by all the students. Students found the communication session on delivering COVID-19 diagnosis particularly helpful.

Overall, most students who completed the post-elective survey (Table 1) strongly or somewhat agreed that the elective met their expectations $(12 / 15,80 \%)$ and was professionally worthwhile $(14 / 15,93 \%)$. Being a VTM allowed them to participate in frontline clinical care $(12 / 15,80 \%)$ and helped them to apply and master their communication skills $(14 / 15,93 \%)$.

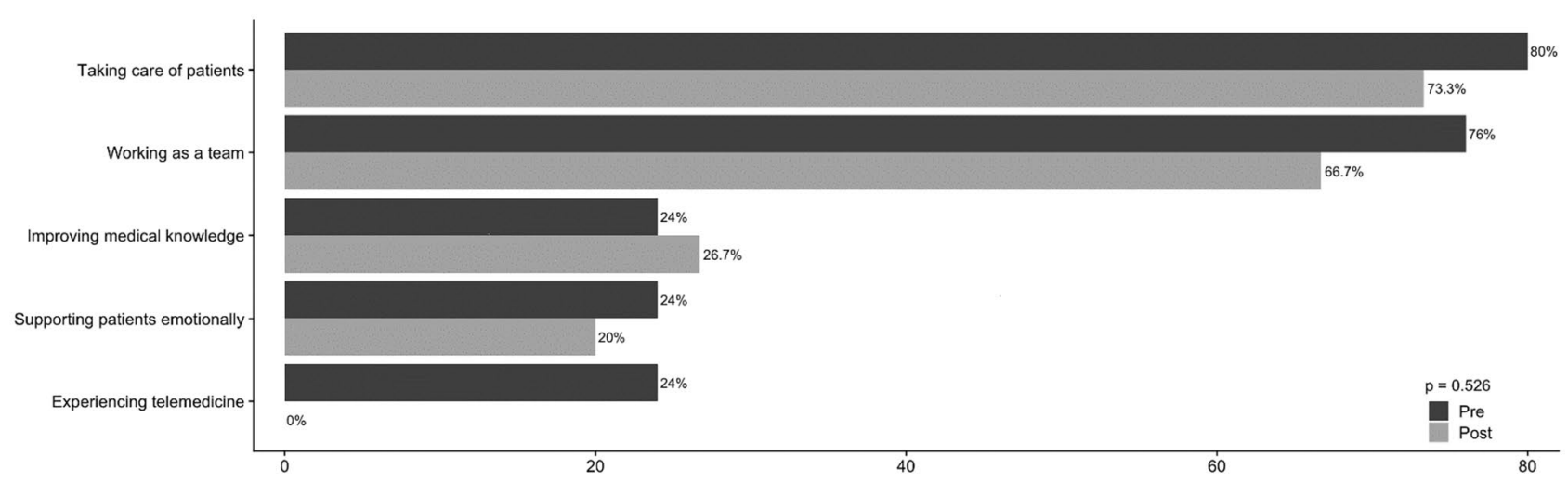

Fig. 2 Comparison of themes between pre-elective expectations and "best part of the elective" Reported on Post-Elective Survey.* *Percentage of keywords manually extracted from the responses to the open-ended questions from pre and post-elective surveys (Student can list multiple answers) 


\section{Discussion}

Despite working remotely, the VTM elective allowed students to learn about COVID-19 care through their direct communication with patients along with virtually shadowing the onsite care teams in the hospital. Such knowledge about a novel, quickly evolving disease is better attained with such contextualized learning and was reinforced by online lecture series. The call by our institutional leaders to continue their medical education also acknowledges the students' unique skill sets in providing a vital role as medically oriented social support for patients and their families. This correlates with students listing taking care of patients as a highly positive experience.

Traditionally situational learning in clerkships blended guided and peripheral participation to develop core competencies. Being the only team member offsite and reliant on the virtual platform raised concerns of disengagement and loss of the students' identity as a member of the care team. Nonetheless, working as a team was frequently cited as the best part of the course. Our efforts to orient and remind the onsite team members to reach out to students throughout the day as well as the students' self-directed engagement contributed to the preservation of the team structure. The VTM elective was rapidly designed to merge academic aspect concerning the safety and continuing education of students with the clinical care front in regard to the urgent need faced by medical teams overwhelmed by the COVID19 pandemic-how do we provide social support and maintain communication with this cohort of emotionally and psychologically vulnerable patients and their families?

The clinical role of medical students during a health crisis can be limited, but removing them entirely from participation deprives them of the opportunity to learn and fulfill their innate sense of duty in contributing to patient care during a historymaking pandemic. Facilitating medical students to participate in roles in which they have been prepared could be beneficial [17]. Patients admitted with COVID-19 are isolated from family and friends, leaving them emotionally and mentally vulnerable. $[18,19]$. Faced with a novel, potentially deadly virus, they can experience fear, loneliness, and despair as well guilt and anxiety for welfare of potentially exposed loved ones. All these emotions may be amplified by physical symptoms such as fevers, weakness, hypoxia, and the uncertainty in prognosis [18]. Comfort from traditional personal encounters, i.e., "hand holding" with patients may also be dampened by the physical barrier posed by personal protective equipment (PPE) donned by healthcare workers, less frequent in-room visits by the team to preserve PPE and minimize exposure, and time constraints imposed on overworked understaffed providers facing rapid clinical fluctuations in their patients. The VTM allowed medical students to use their knowledge and availability to successfully fill that void.
The majority of students agreed that the VTM elective allowed them to take part in patient care and rejoin the frontlines as a core member of the medical team, contributing clinically through telemedicine platforms (phone and video when available) by providing both medical and social support to patients and families, reinforcing clinical information, and playing an integral part in sustaining the flow of communications with the primary providers.

Ensuring consistent experiences for students is always difficult on clinical rotations but was amplified during the VTM. Elective leadership solicited feedback from students throughout the elective with debrief sessions and at the 2 -week point with a mid-elective survey. In addition, format and scheduled activities were adjusted based on student comments following block 1. Lectures were condensed to allow for more time with clinical teams, attending schedules were tracked to catch turnover in team structure, and introduction and expectation emails were sent to all new team members as reminders to increase VTM engagement. Unfortunately, due to the small number of block 2 post-elective survey responses, we could not compare if satisfaction with the course improved with changes implemented from block 1 student feedback.

Given that the elective was also largely focused on meeting an emergent patient care need, our surveys were not designed to be a formal assessment of the course nor of the students' performance but rather to provide continuous quality improvement to optimize the students' experience in real time. Using the data obtained from the free text openended responses, we were able to extract key words, bundled into themes to assess positive and negative aspects of the course, thereby giving only limited appraisal of all the learning objectives. Although the elective fulfilled most students' expectations and was professionally worthwhile highlighting patient care and teamwork, we could not further systematically explore in detail what the specific components were within those themes due to the incomplete survey response rate. The surveys were strongly encouraged but not mandatory, and a fair portion did not complete the post-elective survey, so we opted to present some of students' comments.

We have identified areas for improvement including broadening access to video conferencing technology for hospitalized patients. Frequent attending turnover to protect frontline providers from prolonged exposure and inconsistent teaching opportunities while on service contributed to negative feedback from a few students. Formalized orientation sessions for attendings and other team members on the role of the VTM and tips on how to engage them may help to standardize the experience. Although didactic sessions on communication skills were given in our elective, formal orientation modules on telemedicine delivery such as nonverbal behavior, i.e., telehealth best practices, could have increased 
student confidence and satisfaction with this type of patient interaction [12, 20-22]. We could have further bolstered the elective by including a dedicated small group virtual round for medical students with volunteer tele-instructors similar to what has been utilized in internal medicine clerkship rotation at another institution [16]. Feedback from the hospitalists was difficult to obtain systematically, due to overwhelming clinical responsibilities. Several attendings did submit ad hoc comments about their students' performances and contributions that were included in their formal evaluations. One attending shared,

"Despite being virtual, [the student] was extremely engaged with both the team and with the patients... taking on significant responsibility, touching base with patients, their families, taking notes [remotely] on rounds while [the onsite team was] gowned up with PPE.... [an] integral and invaluable member of the team. On more than one occasion I spoke with family members who wanted to express their appreciation that [the student] was so helpful." Another attending stated that his student was "more than exceptional.... I've been very impressed with her work ethic, commitment to learning and independent thinking. She has been exceptional during rounds...looking up papers, reading articles and talking with patients using iPads and FaceTime with Pacific Interpreters. She has really been a part of the team...."

Depending on the location, the impact of COVID-19 continues to vary widely among AAMC-member medical schools with geographic containment seen but also simultaneous outbreaks and surges in cases across the country and the world where regions in south-east Asia and South America are being disproportionately affected during this spring resurgence. Medical students across the world have been placed in a challenging situation where the disruption of valuable clinical education adds to the sense of inadequate preparedness as future healthcare providers [23-25]. Telemedicine has been increasingly adopted during the pandemic and will likely have a permanent place in the future of healthcare [26]. This virtual care model has quickly been incorporated in clinical rotations for the student learner in various courses [12, 27-29]. Medicine and emergency room clerkships have used telehealth visits to create a safe learning environment while assisting in outpatient care under resident and faculty supervision [12, 30-32]. Inpatient care has been re-imagined virtually in the ICU and subspecialty consult services although geared towards post-clerkship upperclassman with their more care-experienced background [33, 34]. The concept of "the virtual ward" for medical students to follow patients remotely has begun in a Swedish hospital [35]. Our VTM elective demonstrates the feasibility of this concept in the inpatient care setting specifically in patients with SARS-CoV2 infections who face unique isolation challenges and gaps in care that can be filled by students with limited clinical background, should there be a resurgence of COVID-19 given unknown impact of variants or other medical crisis requiring similar interventions. Rapid conversion and implementation to virtual format for medical students may even be possible in resource-limited settings since mobile cell phone technology is now universally readily accessible. Students can remotely engage and experience firsthand the unpredictable clinical course of patients with novel infections in the hospital setting. Patient care and working with the inpatient team were the most frequently cited positive facets of the course, meeting their expectations, and being worthwhile. This "frontline" experience of providing acute care for COVID-19 patients during a global crisis will have a formative impact on their professional identity and future practice.

Supplementary Information The online version contains supplementary material available at https://doi.org/10.1007/s40670-021-01422-8.

\section{Declarations}

Ethics Approval According to institutional review board (IRB) policies at our institution, this work met criteria for quality improvement activities to evaluate and improve a specific practice or program and was exempt from IRB review. All survey data was anonymously collected.

Competing Interests The authors declare no competing interests.

\section{References}

1. Important guidance for medical students on clinical rotations during the Coronavirus (COVID-19) outbreak. News release. AAMC. 2020. Accessed March 1, 2021. https://www.aamc.org/newsinsights/pressreleases/important-guidance-medical-studentsclinical-rotations-during-coronavirus-covid-19-outbreak.

2. Safdieh JE, et al. Curricular response to COVID-19: real-time interactive telehealth experience (RITE) program. Med Educ Online. 2021;26:1918609.

3. Orlando JF, et al. Systematic review of patient and caregivers' satisfaction with telehealth videoconferencing as a mode of service delivery in managing patients' health. PLoS One. 2019;14:e0221848.

4. Vosburg RW, Robinson KA. Telemedicine in primary care during the COVID-19 pandemic: provider and patient satisfaction examined. Telemed J E Health. 2021. https://doi.org/10.1089/tmj.2021.0174.

5. Smrke A, et al. Telemedicine during the COVID-19 pandemic: impact on care for rare cancers. JCO Glob Oncol. 2020;6:1046-51.

6. Koziatek CA, et al. Assessing the impact of a rapidly scaled virtual urgent care in New York City during the COVID-19 pandemic. J Emerg Med. 2020;59:610-8.

7. Ramaswamy A, et al. Patient satisfaction with telemedicine during the COVID-19 pandemic: retrospective cohort study. J Med Internet Res. 2020;22:e20786

8. Tenforde AS, et al. Telemedicine during COVID-19 for outpatient sports and musculoskeletal medicine physicians. PM R. 2020;12:926-32. 
9. Rosen CS, et al. Virtual mental health care in the Veterans Health Administration's immediate response to coronavirus disease-19. Am Psychol. 2021;76:26-38.

10. Murphy RP, et al. Virtual geriatric clinics and the COVID-19 catalyst: a rapid review. Age Ageing. 2020;49:907-14.

11. Pettitt-Schieber B, et al. Implementation and evaluation of eight virtual surgical electives for medical students during the COVID19 pandemic. Am J Surg. 2021. https://doi.org/10.1016/j.amjsurg. 2021.01.032.

12. Abraham HN, et al. Engaging third-year medical students on their internal medicine clerkship in telehealth during COVID-19. Cureus. 2020;12:e8791.

13. Wendt $S$, et al. A virtual COVID-19 ophthalmology rotation. Surv Ophthalmol. 2021;66:354-61.

14. DeVaro SN, et al. Ophthalmology education in COVID-19: a remote elective for medical students. J Acad Ophthalmol. 2020;12:e165-70.

15. Divatia H, Friedland AR. Virtual med-peds: description of the first virtual med-peds student elective during COVID-19. Cureus. 2020;12:e11971.

16. Sukumar $\mathrm{S}$, et al. Designing and implementing a novel virtual rounds curriculum for medical students' internal medicine clerkship during the COVID-19 pandemic. MedEdPORTAL. 2021;17:11106.

17. O'Byrne L, et al. Medical students and COVID-19: the need for pandemic preparedness. J Med Ethics. 2020;46:623-6.

18. Epstein D, et al. Anxiety and suicidality in a hospitalized patient with COVID-19 infection. EJCRIM. 2020;7:001651.

19. Hossain MM, et al. Mental health outcomes of quarantine and isolation for infection prevention: a systematic umbrella review of the global evidence. Epidemiol Health. 2020;42:e2020038.

20. Newcomb AB, et al. Building rapport and earning the surgical patient's trust in the era of social distancing: teaching patientcentered communication during video conference encounters to medical students. J Surg Educ. 2021;78:336-41.

21. Anderi $\mathrm{E}$, et al. Learning communities engage medical students: a COVID-19 virtual conversation series. Cureus. 2020;12:e9593.

22. Rios IC, et al. Virtual interviews between medical students and inpatients during COVID-19 pandemic. Med Educ. 2021;55(5):663.

23. Bongomin $\mathrm{F}$, et al. Internal medicine clerkship amidst COVID19 pandemic: a cross-sectional study of the clinical learning experience of undergraduate medical students at Makerere University. Uganda Adv Med Educ Pract. 2021;12:253-62.

24. Klasen JM, et al. The storm has arrived: the impact of SARSCoV-2 on medical students. Perspect Med Educ. 2020;9:181-5.

25. Alsoufi A, et al. Impact of the COVID-19 pandemic on medical education: medical students' knowledge, attitudes, and practices regarding electronic learning. PLoS One. 2020;15:e0242905.

26. Ahmed S, et al. Telemedicine takes centre stage during COVID-19 pandemic. BMJ Innov. 2020. https://doi.org/10. 1136/bmjinnov-2020-000440.

27. Chandra S, et al. Zooming-out COVID-19: virtual clinical experiences in an emergency medicine clerkship. Med Educ. 2020. https://doi.org/10.1111/medu.14266.

28. Dedeilia A, et al. Medical and surgical education challenges and innovations in the COVID-19 era: a systematic review. In Vivo. 2020;34:1603-11.

29. Chao TN, et al. Creation of an interactive virtual surgical rotation for undergraduate medical education during the COVID-19 pandemic. J Surg Educ. 2020. https://doi.org/10.1016/j.jsurg.2020. 06.039 .

30. Pham DQ, et al. The impact of telehealth on clinical education in adolescent medicine during the COVID-19 pandemic: positive preliminary findings. Front Pediatr. 2021;9:642279.

31. Ren R, et al. Emergency medicine clerkship director experience adapting emergency remote learning during the onset of COVID19 pandemic. AEM Educ Train. 2021. https://doi.org/10.1002/ aet2.10594.

32. Weber AM, et al. An outpatient telehealth elective for displaced clinical learners during the COVID-19 pandemic. BMC Med Educ. 2021;21:174.

33. Ho J, et al. Developing the eMedical Student (eMS)-a pilot project integrating medical students into the Tele-ICU during the COVID19 pandemic and beyond. Healthcare (Basel). 2021;9.

34. Kopp AR, et al. Medical student remote eConsult participation during the COVID-19 pandemic. BMC Med Educ. 2021;21:120.

35. Robertson J, et al. [The virtual ward - the students' clinical education during the current pandemic]. Lakartidningen. 2020;117.

Publisher's Note Springer Nature remains neutral with regard to jurisdictional claims in published maps and institutional affiliations. 